37: 31-34.

SANZ-ELORZA M., E.D. DANA y E. SOBRINO -2004- Atlas de las Plantas Alóctonas Invasoras de España. Ministerio de Medio Ambiente. Dirección General para la biodiversidad. Madrid, $378 \mathrm{pp}$.

URRUTIA, P. -1999- Hydrocharitaceae Juss. In Aizpuru, I. et al. Claves Ilustradas de la Flora del País Vasco y territorios limitrofes: 596-597. Serv. Central Public. Gob. Vasco. 834 pp.

Dirección de los autores: ${ }^{1}$ Agencia Andaluza del Agua. Dirección General del Dominio Público Hidráulico. Plaza de España, Sector II. 41071. Sevilla. ${ }^{2}$ TRAGSA Andalucía. C/Parsi 5, s/n. Pol. PARSI. 41016. Sevilla. *Autor para correspondencia: zcasero@tragsa.es

\title{
148. APORTACIONES AL CONOCIMIENTO DE LA FLORA HALÓFILA DE ANDALUCÍA (S. ESPAÑA)
}

Ma Lucía LENDÍNEZ*, Francisco M. MARCHAL, Juan QUESADA y Carlos SALAZAR

Recibido el 9 de junio de 2009, aceptado para su publicación el 10 de julio de 2009

Publicado "on line" en julio de 2009

\section{Contributions to knowledge on halophilic flora in Andalusia (S. Spain)}

Palabras claves: Corología, fitogeografía, halófitos, Península Ibérica.

Key words: Chorology, phytogeography, halophytes, Iberian Peninsula

Como resultado del estudio de la flora de los ecosistemas halófilos de Andalucía, se muestran 13 taxones halófitos de los cuales 9 constituyen primeras citas provinciales, 1 es citado por primera vez en Andalucía, 2 son primeras citas para Andalucía Oriental, y 6 amplían considerablemente su área de distribución fitogeográfica conocida. Otras 3 citas provinciales, aún no siendo totalmente novedosas, se han incluido en este trabajo por refrendar con pliego de herbario y localidad exacta su presencia en algunas provincias andaluzas.
Los pliegos de referencia a los que corresponden estas citas, se encuentran depositados en las instalaciones de los herbarios de las universidades de Granada (GDA) y/o Jaén (JAEN).

La nomenclatura empleada ha sido la establecida por Flora iberica (Castroviejo et al., 1986-2009) para aquellas familias botánicas actualmente publicadas, y en su defecto, la utilizada en Flora Europaea (Tutin et al., 1972-1980).

Para la comprobación del área ocupada por los taxones citados se ha usado la información 
aportada por Flora iberica, y las bases de datos del Proyecto Anthos (http://www.anthos.es) y Global Biodiversity Information Facility in Spain (GBIF) (http://www.gbif.es), así como las diversas revisiones y notas corológicas existentes en la bibliografía.

De cada una de las especies se indican provincia y localidad de herborización, coordenadas UTM, altitud, fecha de recolección, número de registro de herbario, colector, determinador y, en su caso, revisor. Además, se hace una breve descripción de su ecología y se mencionan las unidades fitogeográficas donde han sido colectadas, siguiendo los criterios de Rivas-Martínez et al. (1997).

\section{Cressa cretica L.}

JAÉN. Higuera de Calatrava. Arroyo Salado. 30SUG9986. 294 m. 05-09-2004. GDA 55078. Leg. \& Det.: M. L. Lendinez \& F. M. Marchal.

Especie halófila que crece en suelos arenosos, arcillosos o salinos, temporalmente inundados de zonas salobres, tanto costeros como continentales. En Andalucía es más frecuente en su parte occidental (http://www.anthos.es), siendo escasa y puntual en las provincias orientales.

Ha sido detectada en diferentes arroyos y lagunas giennenses, en el distrito Hispalense (sector Hispalense, provincia Bética), y constituye la primera cita para la provincia de Jaén. Las localidades más cercanas que se conocen de este taxon, se hallan en la vecina provincia de Córdoba (Puente Genil, Laguna de Tíscar. 30SUH34. 19-06-2002. MGC 52520. Leg. \& Det.: J.M. Nieto, J.L. Díaz, R.M. Conde \& O.M. Comino) y en la provincia de Málaga en las lagunas de Camuñas, Salada de Campillos y Capacete (Martínez Parras, 1984).

Elymus pungens subsp. campestris (Godr. \& Gren.) Melderis

JAÉN. Jaén. Salinas de S. Luis y Sta. Catalina. 30SVG4290. 470 m. 10-06-2004. GDA 55090. Leg. \& Det.: M. L. Lendinez \& F. M. Marchal; Torredonjimeno. Salinas de S. José. 30SVG1179. 449 m. 1906-2004. GDA 55089. Leg. \& Det.: M. L. Lendinez \& F. M. Marchal; Bedmar. Salinas del Tío Pacho. 30SVG6185. 520 m.17-07-2004. GDA 55091. Leg. \& Det.: M. L. Lendinez \& F. M. Marchal.

Especie propia de pastizales xerófilos sobre suelos salinos o yesíferos.

Hasta ahora su presencia en Andalucía era conocida únicamente en Granada, en el distrito Guadiciano-Bastetano, sector Guadiciano-Bacense, provincia Bética, en los ríos Fardes (Fonelas, 30SVG8541, GDAC 40866), Salado del Margen (Cúllar, 30SWG3765, GDAC 40910) y Baza (Baza, GDAC 40911). Con nuestra aportación se amplía su areal fitogeográfico conocido hasta el sector Hispalense. Asimismo, se trata de la primera cita refrendada con pliego de herbario para la provincia de Jaén, en la que consta una antigua cita algo dudosa en el término de Cazorla (Fernández Galiano \& Heywood, 1960), de la que no se detalla la subespecie ni se aporta pliego de herbario. Además, existe otra cita en las sierras de Jaén, que posteriormente ha resultado ser errónea (Fernández López \& Amezcua, 1986; Vieira \& Fernández López, 1989).

\section{Frankenia laevis L.}

JAÉN. Santiago de Calatrava. Proximidades arroyo Salado de Porcuna. 30UG9878. 317 m. 0509-2004. GDA 55079. Leg. \& Det.: M. L. Lendinez \& F.M. Marchal.

Planta halófila o halonitrófila que se desarrolla en suelos salinos, arenosos o limosos, poco húmedos y frecuentemente algo nitrificados. Se trata de una especie con una mayor presencia en zonas litorales. La localidad continental más cercana se encuentra en la provincia de Granada, concretamente en los saladares de La Malahá (Esteve \& Varo, 1975).

Se ha herborizado en un arroyo del distrito Hispalense (sector Hispalense, provincia Bética), donde constituye la primera cita para la provincia de Jaén.

\section{Gypsophila tomentosa L.}

GRANADA. La Malahá. Llano junto rotonda hacia Ventas de Huelma. 30SVG3505. 750 m. 18-062007. GDA 54240. Leg. \& Det. F. M. Marchal.

Especie que se desarrolla sobre suelos salinos $\mathrm{y}$ húmedos, generalmente en depresiones saladas y ocasionalmente en bordes de caminos, formando parte de praderas de saladillos (Limonium sp. pl.).

Hasta ahora sólo se conocía su presencia en zonas endorreicas de Castilla y León, Castilla-La Mancha, valle del Ebro, Comunidad Valenciana y Hoya de Guadix-Baza (Granada), siendo esta última la única localidad andaluza donde se había observado [Esteve Chueca \& Varo, 1975 (sub G. perfoliata); Gamarra \& Montouto, 1994; Salazar et al., 2002]. 
Recientemente la hemos colectado en las proximidades de los saladares interiores de La Malahá (Granada), en el distrito Alfacarino-Granatense (sector Malacitano-Almijarense, provincia Bética), lo que supone una interesante ampliación del areal fitogeográfico conocido de este taxon.

En la comunidad autónoma andaluza, se considera una especie de gran interés, que actualmente está catalogada como especie Vulnerable en la Lista Roja de la Flora Vascular de Andalucía (Cabezudo et al., 2005).

Hymenolobus procumbens (L.) Nutt. subsp. procumbens

JAÉN. Mancha Real. Arroyo La Pilera, Salinas de "El Sali". 30SVG4797. 375 m. 21-03-2003. GDA 55080. Leg. C. Salazar, M.L. Lendínez, A. Sevilla \& F. Guerrero. Det: M.L. Lendinez. \& F. M. Marchal.

Taxon que aparece sobre sustratos salinos húmedos tanto costeros como continentales, aunque también aparece sobre calizas, arenas o en medios algo nitrificados.

Recientemente ha sido herborizado en el distrito Hispalense (sector Hispalense, provincia Bética) de Jaén. En esta provincia sólo se conocía la existencia de la subespecie pauciflorus, propia de roquedos calizos de montaña (Fernández López \& Morillas Sánchez, 1994), por lo que la presencia de la subespecie tipo supone su primera cita provincial.

\section{Limonium ovalifolium (Poir.) Kuntze}

SEVILLA. Lebrija. Arroyo de las Salinas. 30STF3787. 78 m. 11-06-2005. GDA 55083. Leg. \& Det.: M. L. Lendínez \& F. M. Marchal.

Especie que se desarrolla en sustratos arenosos, ricos en sales. En la Península Ibérica sus poblaciones son muy escasas, conociéndose únicamente de localidades correspondientes al Algarve, Cantabria, Vizcaya, Huelva y Cádiz.

Recientemente ha sido herborizada en el interior de Andalucía, concretamente en el valle del Guadalquivir en Sevilla (distrito Hispalense, sector Hispalense, provincia Bética), donde constituye la primera cita provincial y una ampliación del área fitogeográfica conocida de la especie desde la costa Gaditano-Onubo-Algarviense hacia zonas más continentales de la provincia Bética.

\section{Lythrum baeticum Gonz.-Albo}

JAÉN. Cazorla. Salina de Las Arcas. 30SVG9002. 444 m. 17-04-2004. GDA 55081. Leg.
\& Det. M.L. Lendinez \& F.M. Marchal.

Especie propia de pastizales terofíticos sobre suelos húmedos, generalmente margosos y en ocasiones salinos. Se trata de un endemismo iberonorteafricano, con escasa distribución en la Península Ibérica y en Andalucía, en la que es conocida su presencia en la porción occidental (Cádiz, Córdoba, Huelva y Sevilla).

Esta especie aparece catalogada como EN, tanto a un nivel nacional en el Atlas y Libro Rojo de la Flora Vascular Amenazada de España (Bañares et al., 2003) y la Lista Roja 2008 de la Flora Vascular Española (Bañares et al. 2008) como autonómico, en la Lista Roja de la Flora Vascular de Andalucia (Cabezudo et al., 2005). En esta última obra aparece citada en la provincia de Jaén, pero sin existir pliegos de herbario que lo confirmaran, por lo que ésta sería la primera cita refrendada con pliego testigo.

La hemos herborizado en el distrito Hispalense (sector Hispalense, provincia Bética). Se ha observado que el material recolectado presenta ciertos caracteres algo intermedios entre $L$. junceum y $L$. baeticum, ya que posee un porte algo rastrero y hojas con mayor tamaño que lo propio en L. baeticum (según lo descrito por Velayos, 1997). Sin embargo, carece de las manchas rojizas en el tubo floral, que caracterizan a $L$. junceum. No se descarta por tanto que ambas especies se hibriden en el territorio de la provincia de Jaén.

Puccinellia hispanica Julià \& J.M. Monserrat

MÁLAGA. Campillos. Laguna Salada. 30SUG3600. 446 m. 01-05-2005. GDA 55076. Leg. \& Det.: M. L. Lendinez \& F. M. Marchal. Rev. C. Salazar \& A.T. Romero; Campillos. Laguna de Capacete. 30SUG3798. 454 m. 01-05-2005. GDA 55077. Leg. \& Det.: M. L. Lendinez \& F. M. Marchal. Rev. C. Salazar \& A.T. Romero; Campillos. Laguna Dulce. 30SUG3601. 455 m. 27-05-2006. GDA 55075. Leg. \& Det.: M. L. Lendinez \& F. M. Marchal. Rev. C. Salazar \& A.T. Romero; Campillos. Laguna de la Marcela. 30SUF3698. 465 m. 27-05-2006. GDA 55074. Leg. \& Det.: M. L. Lendinez \& F. M. Marchal. Rev. C. Salazar \& A.T. Romero.

Especie propia de suelos arcillosos salinos, algo nitrificados y temporalmente encharcados de lagunas salobres, cuencas endorreicas y llanuras del interior. Generalmente aparece en ambientes secos o semiáridos, donde forma densos céspedes casi monoespecíficos.

Se trata de un endemismo iberonorteafricano 
escasamente representado en la Península Ibérica (Prepirineo, depresiones del Ebro y del Duero, Sistema Ibérico, Castilla-La Mancha, Valencia y Andalucía Occidental). En Andalucía solamente es conocida su existencia en la laguna del Gosque, provincia de Sevilla (Juliá, 1992). Recientemente la hemos recolectado en diferentes lagunas del complejo endorreico de Campillos, por lo que nuestra aportación supone la primera cita para la provincia de Málaga, y por extensión para toda Andalucía Oriental.

Se trata de una especie de aspecto muy similar a P. fasciculata (Torrey) E.P. Bicknell, con la que ha podido ser confundida hasta el momento. Los ejemplares recolectados en la provincia de Málaga presentan algunos caracteres intermedios entre ambas especies. Así, el número de costillas de las hojas del material herborizado se asemeja más al de P. fasciculata (Juliá \& Montserrat, 1999) mientras que el tamaño de las anteras y las lemas se ajustan mejor a las dimensiones de $P$. hispanica.

La presencia de $P$. fasciculata en dicho complejo endorreico está refrendada por diversos pliegos de herbario (MGC 52607, 52614, 55957 y 9031).

\section{Puccinellia tenuifolia G. Monts. \& J.M. Monts.}

MÁLAGA. Fuente de Piedra. Laguna de Fuente de Piedra. 30SUG4511. 412 m. 27-05-2006. GDA 55092. Leg. \& Det.: M. L. Lendínez \& F. M. Marchal. Rev. C. Salazar \& A.T. Romero.

Especie propia de marismas y zonas salobres, temporalmente encharcadas y en ocasiones nitrificadas.

Se trata de una especie iberonorteafricana que se encuentra puntual y escasamente representada en el suroeste de la Península Ibérica. Hasta ahora su existencia en Andalucía estaba sólo probada en el litoral atlántico de las provincias de Huelva y Cádiz (sector Gaditano-Onubense, provincia GaditanoOnubo-Algarviense). La presencia de esta especie en la laguna hipersalina de Fuente de Piedra (distrito Hispalense, sector Hispalense, Provincia Bética), supone la primera cita refrendada con pliego de herbario para la provincia de Málaga y para Andalucía Oriental. Asimismo, constituye una importante ampliación del areal fitogeográfico conocido de este taxon, desde la provincia Gaditano-Onubo-Algarviense hasta la Bética.

Puccinellia tenuifolia había sido herborizada con anterioridad en esta misma área, como lo atestiguan diversos pliegos de herbario (GDAC 5043,
MGC 5024, 5025, 8090, 8091, 9025 y 47038), aunque no había sido publicada. Por otra parte, Martínez Parras (1984) cita P. festuciformis subsp. tenuifolia en las lagunas de Capacete, Cerero, Fuente de Piedra, Ratosa y Salada de Campillos (Málaga) y la laguna del Conde (Córdoba), pero no aporta pliegos de herbario, por lo que a juzgar por la abundancia del taxon en sus inventarios, opinamos que podría tratarse de $P$. fasciculata, muy frecuente en el referido territorio.

\section{Salicornia ramosissima Wood}

GRANADA. Cúllar, El Margen. Salado del Margen. 30SWG3366. 794 m. 22-04-2006. GDA 55085. Leg. \& Det. M.L. Lendinez \& F. M. Marchal.

Terófito halófilo que se desarrolla en suelos salinos de lagunas salobres y saladares temporalmente encharcados, tanto del litoral como del interior.

Herborizado en la cuenca del Guadiana Menor (distrito Guadiciano-Bastetano, sector GuadicianoBacense, provincia Bética), constituye la primera cita provincial para Granada, ya que se tenía constancia de su presencia en las siete provincias restantes de Andalucía. Las poblaciones más cercanas se sitúan en varias localidades de la provincia de Jaén (Casado \& Fernández López, 1988).

\section{Suaeda pruinosa Lange}

JAÉN. Quesada. Rambla del Vilano. 30SVG8486. 420 m. 11-06-1999. GDA 54219. Leg. \& Det. F. M. Marchal; Huesa. Salina El Salar. 30SVG8980. 430 m. 23-07-2004. GDA 55086. Leg. \& Det. M.L. Lendinez \& F. M. Marchal.

Especie halonitrófila que aparece en zonas nitrificadas y salinas de tendencia árida, generalmente en territorios litorales del sureste peninsular.

En Andalucía su presencia se había indicado para las provincias de Almería y Granada (Alonso \& De la Torre, 2003). Con su herborización en la cuenca del Guadiana Menor (distrito Guadiciano-Bastetano, sector Guadiciano-Bacense, provincia Bética), se aporta la primera cita para la provincia de Jaén.

\section{Tamarix boveana Bunge}

GRANADA. Cúllar, El Margen. Salado del Margen. 30SWG3366. 795 m. 29-04-2007. GDA 52980, JAEN 670427. Leg. \& Det. M. L. Lendínez \& F. M. Marchal. Rev. C. Salazar; Cúllar. Rambla de Maciamolá. 30SWG3463. 824 m. 25-07-2007. GDA 54257. Leg. \& Det. F. M. Marchal. 
Taxon que crece sobre suelos húmedos $\mathrm{y}$ salinos, bordes de arroyos, ramblas, depresiones encharcadas y zonas arenosas próximas al litoral. Se ha herborizado en la cuenca del Guadiana Menor (distrito Guadiciano-Bastetano, sector GuadicianoBacense, provincia Bética).

Se trata de una cita de gran importancia puesto que este taxon iberonorteafricano se encuentra puntual y escasamente representado en el este de la Península Ibérica y Baleares. En Andalucía solamente era conocido en algunas zonas costeras de la provincia de Almería (Molina et al., 1990), donde constituye tarayales hiperhalófilos del Inulo crithmoidis-Tamaricetum boveanae Izco, FernándezGonzález \& A. Molina 1984

La presencia de esta especie en el interior de Andalucía (probablemente la localidad a mayor altitud hasta ahora reportada), supone la primera cita en la provincia de Granada, y una importante ampliación de su areal fitogeográfico conocido, que aumenta desde la provincia Murciano-Almeriense hasta la Bética. De esta manera se constata la presencia de dichos tarayales hiperhalófilos, que actuarían como cabeza de serie de la vegetación hiperhalófila guadiciano-bastetana, hasta ahora no detectada (Salazar et al., 2002).

\section{Tamarix dalmatica Baum}

ALMERÍA. Berja. Río Chico. 30SWF0071. 76 m. 24-02-2007. GDA 52981, JAEN 70428. Leg. \& Det.: M. L. Lendinez, F. M. Marchal \& J. Quesada. Rev. C. Salazar.

Especie halófila que aparece sobre suelos húmedos, salinos o carbonatados, en bordes de arroyos, ramblas o vaguadas.

Se trata de una cita de gran importancia, puesto que este taxon se encuentra puntualmente representado en la Península Ibérica (Levante e Islas Baleares) y hasta la actualidad no era conocido en Andalucía.

Ha sido herborizado en el distrito Almeriense Occidental (sector Almeriense, provincia MurcianoAlmeriense), por lo que supone la primera cita para esta provincia y para toda Andalucía, así como una ampliación del areal fitogeográfico conocido de la especie, desde el sector Murciano al Almeriense.

AGRADECIMIENTOS. Agradecemos a la Dra. Ana Teresa Romero (Departamento de Botánica de la Universidad de Granada) la revisión de los pliegos testigo del género Puccinellia.

\section{BIBLIOGRAFÍA}

ALONSO, M. A. y A. DE LA TORRE -2002- Las comunidades fruticosas de Suaeda Forsskål ex J. F. Gmelin en la Península Ibérica e islas Baleares. Lazaroa 23: 95-105.

BAÑARES, Á., G. BLANCA, J. GÜEMES, J.C. MORENO y S. ORTIZ (EDS.) -2003-Atlas y Libro Rojo de la Flora Vascular Amenazada de España. Táxones Prioritarios. Ministerio de Medio Ambiente. Madrid.

BAÑARES, Á., G. BLANCA, J. GÜEMES, J.C. MORENO y S. ORTIZ (EDS.) -2008- Lista Roja 2008 de la Flora Vascular Española. Ministerio de Medio Ambiente y Medio Rural y Marino. Madrid.

CABEZUDO, B., S. TALAVERA, G. BLANCA, C. SALAZAR, M. CUETO, B. VALDÉS, J.E. HERNÁNDEZ-BERMEJO, C. HERRERA, C. RODRÍGUEZ-HIRALDO y D. NAVAS -2005- Lista Roja de la Flora Vascular de Andalucía. Consejería de Medio Ambiente. Junta de Andalucía. Sevilla.

CASADO PONCE, D. y C. FERNÁNDEZ LÓPEZ -1988-Chenopodiáceas, Amarantáceas, Portulacáceas y Aizoáceas de la provincia de Jaén. Blancoana 6: 37-52.

CASTROVIEJO, S. ET AL. (EDS.) -1986/2009Flora iberica. vols. I-VIII, X, XII-XV, XVIII y XXI. Real Jardín Botánico. C.S.I.C. Madrid.

ESTEVE, F. y J. VARO -1975- Estudio geobotánico de las comunidades halófilas interiores de la provincia de Granada. Anales Inst. Bot. Cavanilles 32 (2): 1351-1374.

FERNÁNDEZ-GALIANO, E. y V. H. HEYWOOD -1960- Catálogo de plantas de la provincia de Jaén (Mitad Oriental). Inst. Est. Giennenses. Jaén.

FERNÁNDEZ LOPEZ, C. y C. AMEZCUA -1986Anotaciones a plantas vasculares poco conocidas en Jaén. II. Blancoana 4: 65-81.

FERNÁNDEZ LOPEZ, C. y L. MORILLAS SÁNCHEZ -1994- Plantas del Guadiana Menor en el herbario JAEN hasta 1993. Blancoana 11: 54-63.

GAMARRA, R. y O. MONTOUTO -1994Adiciones. Mapa 422. Fontqueria 40: 102.

JULIÁ, M. A. -1992- El gènere Puccinellia Parl. (Poaceae) a la Peninsula Iberica. Collecció 
de Tesis Doctorals microfitxades, núm. 1462. Universitat de Barcelona.

JULiÁ, M. A. y J. M. MONTSERRAT -1999Dos táxones nuevos en el género Puccinellia (Poaceae). Fontqueria 53: 3-4.

MARTÍNEZ PARRAS, J.M. -1984- La vegetación lacustre de la depresión de Antequera (Andalucía). Collect. Bot. (Barcelona) 15: 289-306.

MOLINA, A., F. FERNÁNDEZ GONZÁLEZ y J. LOIDI -1990- Cartografía Corológica Ibérica. Aportación 11. Bot. Complut. 16: 132-133.

RIVAS-MARTÍNEZ, S., A. ASENSI, B. DÍEZGARRETAS, J. MOLERO-MESA \& F. VALLE -1997- Biogeographical synthesis of Andalusia (southern Spain). J. Biogeography 24: 915928.

SALAZAR, C., J.A. TORRES, F.M. MARCHAL y

E. CANO-2002-. La vegetación edafohigrófila del distrito Guadiciano-Bastetano (GranadaJaén, S. España). Lazaroa 23: 45-64.

TUTIN, T.G., V.H. HEYWOOD, N.A. BURGES, D.M. MOORE, D.H. VALENTINE, S.M. WALTERS \& D.A. WEBB (EDS.) -1964-1980Flora Europaea, vols. 1-5. Cambridge Univ. Press, Cambridge.

VELAYOS, M. -1997- Lythrum L. In: Castroviejo, S.; C. Aedo; C. Benedía; M. Laínz; F. MuñozGarmendia, G. Nieto Feliner \& J. Paiva (eds.) Flora iberica. vol. VIII HaloragaceaeEuphorbiaceae. Real Jardín Botánico. C.S.I.C. Madrid.

Dirección de los autores. Departamento de Biología Animal, Biología Vegetal y Ecología. Facultad de Ciencias Experimentales. Edif. B3. Universidad de Jaén. Paraje Las Lagunillas s/n. 23071-Jaén. *Autora para correspondencia: lulendi@ujaen.es

\title{
149. APORTACIONES A LA FLORA DE ANDALUCÍA OCCIDENTAL
}

\author{
Carlos LUCENA, Félix INFANTE y Emilio RUIZ DE CLAVIJO*
}

Recibido el 8 de mayo de 2009, aceptado para su publicación el 13 de mayo de 2009 Publicado "on line" en mayo de 2009

New contribution on the flora of western Andalusia

Palabras clave. Flora, corología, Córdoba, Andalucía.

Key words. Flora, chorology, Cordoba, Andalusia.

La construcción del Embalse La Breña II en el término municipal de Almodóvar del Río (Córdoba) ha implicado la realización de una serie de medidas compensatorias, una de las cuales ha sido el estudio de la flora que vive en los márgenes del actual embalse La Breña, y en

Trabajo realizado con cargo al proyecto "Medidas Compensatorias del Embalse de la Breña II. Estudio sobre la Flora Amenazada", financiado por Ingeniería y Gestión del Sur/ IPA S.L. 